\title{
Propuesta de un modelo de co-gestión para los Pequeños Abastos Comunitarios de Agua en Colombia
}

\author{
Andrea Bernal, Luis Rivas y Pilar Peña*
}

\section{Resumen}

Este artículo recopila la evolución conceptual de la gestión comunitaria a partir de diferentes perspectivas: capital social, bienes comunes y co- gestión o gestión colaborativa y presenta las principales estrategias de co-gestión aplicadas al abastecimiento de agua: el modelo de desarrollo local conducido por la comunidad y la localidad o LCDD y el modelo consensuado de suministro o Triple S. Con este sustento teórico, se propone un modelo conceptual de co-gestión para pequeños abastos comunitarios de agua en Colombia, modelo de enfoque territorial y funcional que incorpora diferentes niveles de gobernanza (comunitario/local, regional y nacional) con funciones articuladas en espacios anidados, para garantizar el acceso al agua potable en comunidades rurales.

\begin{abstract}
This paper reviews the conceptual evolution of Community Based Monitoring (CBM) from different approaches : social capital, common pool resources and co-management or collaborative management, and presents the main co-management strategies applied to water supply at small scale: local and community driven development (LCDD) and sustainable services at scale (SSS). Supported by this theoretical background, and in order to improve access to drinking water in rural communities in Colombia, the authors propose a co-management model for small and community water supplies.
\end{abstract}

Palabras clave: pequeños abastecimientos de agua, gestión comunitaria del agua, bienes comunes, co-gestión, gobernanza del agua, gestión colaborativa, modelos de co-gestión. Key words: Small Community Water Supply (SCWS), Community-Based Management (CBM), Common-Pool Resources (CPR), Local and Community Driven Development (LCDD), multi-level governance.

* Andrea Bernal es maestra en Dirección y Gerencia de Empresas, candidata a doctora en Ciencias Administrativas en la Escuela Superior de Administracion (ESCA) Santo Tomás del Instituto Politécnico Nacional de México (IPN). Ha sido docente de la Universidad del Rosario en Bogotá, Colombia y Consultora del Banco Interamericano de Desarrollo (BID) en temas de abastecimiento rural de agua y saneamiento. Actualmente realiza estudios en desarrollo sostenible en la Universidad de Exeter, Reino Unido.

Luis Rivas es doctor en Ciencias Administrativas y en Estudios Europeos. Es catedrático de la ESCA Santo Tomás del IPN e Investigador Nacional.

Pilar Peña es Doctora en Ciencias Administrativas, catedrática de la ESCA Santo Tomás del IPN e Investigadora Nacional. 


\section{Introducción}

a gestión comunitaria es una de las formas tradicionales de administración de los recursos naturales en comunidades indígenas y campesinas (Palerm y Martínez, 2009) siendo parte de la identidad socio-cultural de muchos pueblos aún observable en áreas rurales y periurbanas de los países en desarrollo (Boelens, 2002). Los pequeños abastos comunitarios de agua o "Small Community Water Supplies (SCWS)", también llamados "acueductos comunitarios" en Colombia, o "Juntas de Agua" en Perú, $\mathrm{u}$ "Organizaciones Comunitarias de Servicios de Agua y Saneamiento (OCSAS)" son sistemas de abasto de agua para consumo humano construidos y operados por las propias comunidades, mediante una organización participativa y con una concepción colectiva de la propiedad. Se estima que existen 80,000 de estas organizaciones en Latinoamérica, surtiendo agua para más de cuarenta millones de personas (AVINA, 2011: 24). En Colombia, más de 12,000 organizaciones comunitarias abastecen de agua aproximadamente a siete millones de personas (Rojas, Tamayo y García, 2011) y son una forma autorizada para la prestación de los servicios básicos de agua y saneamiento. Pese a ello, aún no se ha desplegado una política pública o un modelo de gestión a nivel nacional.

La gestión comunitaria ha sido objeto de análisis teórico sólo en las cuatro últimas décadas (Binswanger-Mkhize, De Regt y Spector, 2009). En los años 70 se inició la observación de la gestión comunitaria como fenómeno rural. En los 80, bajo el enfoque del capital social (iniciativa auspiciada por el Banco Mundial) la gestión comunitaria fue promovida como un escenario de fortalecimiento del tejido social (Feldman y Assaf, 1999), tendencia que continúa siendo aplicada en los enfoques de empoderamiento. En los 90, a partir de la teoría de los bienes comunes se cuestiona el arquetipo de la economía clásica sustentado en la interacción individuo-sociedad-estado en un ambiente competitivo, para presentar nuevas alternativas de gobernanza apoyadas en el reconocimiento de la comunidad como colectividad colaborativa y en la posibilidad de la interacción humana bajo reglas de cooperación (Ostrom, 1990). Desde mediados de los años 90, la gestión colaborativa o co-gestión recupera el valor de la interacción entre los actores o stakeholders en el que los individuos (como expresión de la iniciativa individual), las comunidades (como expresión de la acción colectiva), los gobiernos locales, regionales y nacionales y otras organizaciones 
de la sociedad civil toman parte, colaborando en estructuras participativas (Carlsson y Berkes, 2005).

Los pequeños abastos comunitarios de agua u OCSAS, son esenciales para satisfacer las necesidades alimentarias y sanitarias de la población rural en Latinoamérica y representan una oportunidad para empoderar a las comunidades y facilitar el desarrollo local (Bernal, 2011). También es deseable que los gobiernos nacionales y locales apoyen la gestión comunitaria desde los diferentes niveles de gobierno, contribuyendo así a la sostenibilidad de los recursos hídricos y al cierre de la brecha en el acceso al agua potable y al saneamiento en áreas rurales (WHO y UNICEF, 2012). Sin embargo, los países en desarrollo apenas han iniciado la formulación de estrategias específicas para la gestión comunitaria del agua y el saneamiento (Lockwood y Smits, 2011) que requieren estar alineadas con las condiciones del contexto local.

A continuación se describirán los diferentes enfoques conceptuales de la gestión comunitaria, para describir los modelos conceptuales de co-gestión que están siendo empleados para el abastecimiento del agua, estableciendo sus variables para proponer un modelo conceptual que nos lleve a una estrategia de apoyo a los acueductos comunitarios en Colombia.

\section{Marco conceptual de la gestión comunitaria}

Si bien las relaciones jurídicas y económicas del mundo occidental se fundan en la dicotomía individuo - Estado, las personas continúan alimentando fuertes relaciones en su comunidad, entendida como grupo de individuos que comparten intereses o expectativas. Para el manejo de los recursos naturales, la comunidad incorpora dimensiones socio-ambientales, como el territorio y la cultura que surgen de una construcción histórica colectiva. Para el abasto de agua dirigido al consumo humano, la comunidad además requiere el apoyo del Estado como garante de la provisión de servicios públicos y es imprescindible la colaboración de individuos y empresas como agentes económicos y ambientales en el aprovechamiento y disposición de los recursos hídricos. Por ello, la gestión del agua por cuenta de las comunidades implica relaciones entre múltiples actores o stakeholders condicionadas por las reglas del gobierno nacional, regional y local.

En este contexto, la gestión comunitaria es una respuesta espontánea y de acción colectiva para satisfacer las necesidades de abastecimiento de agua en una localidad, y puede entenderse como un modelo de gestión autónomo bajo el cual una comunidad se organiza y establece sus propias 
reglas para el aprovechamiento del recurso (Lockwood, 2002). Por otra parte, la co-gestión o gestión colaborativa, es un modelo de gestión heterónomo en el que interviene el Estado desde el gobierno central y local, el sector privado, las comunidades y la sociedad civil (The World Bank, 1999). Así la diferencia entre la gestión comunitaria y la co-gestión radica en el número y naturaleza de los actores o involucrados clave. La co-gestión es una forma más compleja de gestión que involucra la colaboración de múltiples sectores y requiere un ejercicio participativo para llegar a consensos. En la co-gestión se observan también niveles intermedios de gobierno como las organizaciones no gubernamentales -ONG's, las asociaciones de organizaciones de base comunitaria, organizaciones privadas (Andersson y Ostrom, 2008)- que fortalecen la gestión comunitaria y el desarrollo local.

\section{La importancia del capital social en la gestión comunitaria}

La primera definición de capital social fue propuesta por Bourdieu (1986: 51) para referirse a "...el agregado de los recursos actuales y potenciales que están vinculados con la posesión de una red durable de relaciones más o menos institucionalizadas de mutuo reconocimiento - en otras palabras, la participación en un grupo- que provee a cada uno de sus miembros con el retorno de capital obtenido colectivamente..." Así, la composición tradicional del capital natural, el capital producido y el capital humano fue ampliada para incluir al capital social, que se refiere a la coherencia interna y cultural de la sociedad, a las normas y valores que gobiernan las interacciones entre las personas y a las instituciones a las cuales estas personas están vinculadas. Feldman y Assaf (1999) recopilaron los antecedentes conceptuales del capital social y un acervo de la evidencia empírica de estudios de caso previos a 1999, demostrando la enorme influencia de la acción social conjunta sobre los recursos naturales.

El rol de la comunidad para el fortalecimiento del tejido social ha sido defendido ampliamente por un gran número de autores: Etzioni (1993) alerta sobre la pérdida de valores civiles en la sociedad americana y exalta el movimiento comunitario desde una perspectiva moral y social. Putnam (1993) sugiere que el capital social puede ayudar a tender puentes entre divisiones ideológicas y ayuda a fortalecer el consenso sobre la importancia del empoderamiento. Woolcock (1998) argumenta que pueden identificarse dos clases de capital social: el nivel micro que surge del emprendimiento étnico y se sostiene mediante las relaciones entre diferentes comunidades y el nivel macro o institucionalismo comparativo que se refiere a la ca- 
pacidad y credibilidad institucional y se fortalece tras la participación en organizaciones más amplias. Grootaert (1998) retoma la discusión sobre la estructura del capital social en el nivel micro y el nivel macro y afirma que ..."las asociaciones e instituciones proveen una estructura informal para organizar el intercambio de información, la coordinación de actividades y la toma de decisiones en la acción colectiva" (citado en Feldman y Assaf, 1999: 78).

La evidencia empírica sobre la gestión comunitaria, sustenta la propuesta conceptual del capital social. Narayan y Pritchett (1997) observaron las características de la actividad asociativa en comunidades rurales de Tanzania, en particular los enlaces entre los individuos en asociaciones voluntarias sumados a las normas cívicas y sociales, como constructores de confianza y de capital social. A partir de estas variables crearon un índice de la vida asociativa en las comunidades rurales, y después compararon este índice con el nivel de ingreso familiar en cada comunidad. La investigación concluyó en que para catalogar el capital social como tal, éste debe incrementar el ingreso de las familias, identificando canales a través de los cuales el capital social incide en el ingreso (esto puede verse reflejado en los servicios públicos mejorados, el uso creciente de conocimientos y tecnologías modernas en la agricultura, mayor actividad comunitaria y uso extendido del crédito en agricultura) (Feldman y Assaf, 1999: 26).

El capital social interpreta a la gestión comunitaria como un retorno a formas tradicionales de organización social, centradas en las comunidades como espacios de interacción social con impacto en el bienestar individual. Sin embargo, esta apreciación no se aparta de los paradigmas de la economía clásica de explotación de los recursos naturales y aprovechamiento egoísta del entorno, entendiendo a la comunidad como un vehículo para la satisfacción de las necesidades individuales. Por otra parte, el reconocimiento de los valores comunitarios desde la perspectiva conceptual y empírica constituye un llamado a la promoción de la gestión comunitaria como escenario para el desarrollo, y por ello numerosas estrategias de acceso al agua se han enfocado en el fortalecimiento de capacidades de la comunidad para la gestión local y el estímulo a la participación colectiva.

Pese a la importancia de la comunidad como escenario para la cohesión social, su comprensión como fenómeno espacial o social es aún limitada. La literatura se refiere indistintamente a las comunidades locales, a los usuarios del recurso, a los residentes locales o a la sociedad civil, sin aclarar las diferencias entre estos conceptos. Así no resulta claro que la comunidad es un actor con identidad propia y una voz común, que surge de la acción colectiva como un proceso complejo de decisión. De hecho, las comunida- 
des son sistemas complejos que no pueden ser caracterizadas de manera unitaria. Además de las diferencias étnicas, culturales, económicas y sociales, las comunidades están en constante cambio y deben ser interpretadas como unidades sociopolíticas multidimensionales (Berkes, 2009). Si bien las aproximaciones antropológica, histórica y social son determinantes para el desarrollo comunitario, desde la perspectiva de las ciencias de la administración es importante establecer las particularidades de la comunidad en su dimensión organizacional (relaciones y toma de decisiones), participativa (influencia en la toma de decisiones) y de gestión (manejo de las iniciativas comunes) (Awortwi, 2012) con el fin de establecer las necesidades de desarrollo de capacidades.

\section{La gestión comunitaria vista desde la teoría de los bienes comunes}

Ostrom (1990), describe los tres modelos más usados para explicar las relaciones económicas entre los individuos y los recursos naturales. El resumen de la crítica a estos modelos puede leerse a continuación:

"El primero de ellos (los modelos) es conocido como "la tragedia de los comunes" el cual se refiere a la degradación del medio ambiente que puede ocurrir cuando muchos individuos usan en común un recurso escaso. El segundo es conocido como el "dilema del prisionero" y se caracteriza porque cada jugador selecciona su estrategia individual más favorable. El tercer modelo es la "lógica de la acción colectiva" , y se enfoca en que los individuos persiguen sus beneficios compartidos más que su bienestar individual. Estos modelos, siguen el supuesto de que los individuos no cooperarán sino para alcanzar los beneficios colectivos, y parecen atrapados en situaciones estáticas sin la habilidad de cambiar las reglas afectando sus incentivos" (Ostrom, 1990, citado en Feldman y Assaf, 1999: 29).

A partir de los cuestionamientos a estos paradigmas, Ostrom (1990) afirma que los individuos están dispuestos a manejar los recursos naturales colectivamente, comprometiéndose en una estrategia cooperativa que ellos mismos diseñan, con fundamento en sus capacidades de autorregulación, autogestión y en el reconocimiento de diferentes niveles de gobernanza. Así las comunidades configuran grupos con autoridad de decisión sobre la gestión de un recurso, coordinan los aportes necesarios y sancionan a quienes no contribuyen y estructuran su nivel de gobierno, formal o informal. 
La teoría de los recursos de uso común cuenta hoy con un modelo conceptual para la comprensión de los sistemas socio-ecológicos complejos (social-ecological-systems - SES) (ver figura 1), que interpreta la gestión de los recursos naturales como un conjunto de sistemas y subsistemas que permite la interdependencia entre los ecosistemas y los aspectos sociales, económicos y políticos que los gobiernan (Ostrom, 2009).

Figura 1. Los principales subsistemas en una estructura para analizar sistemas socio-ecológicos (SES)

(S) ASPECTOS SOCIALES, ECONOMICOS Y POLITICOS

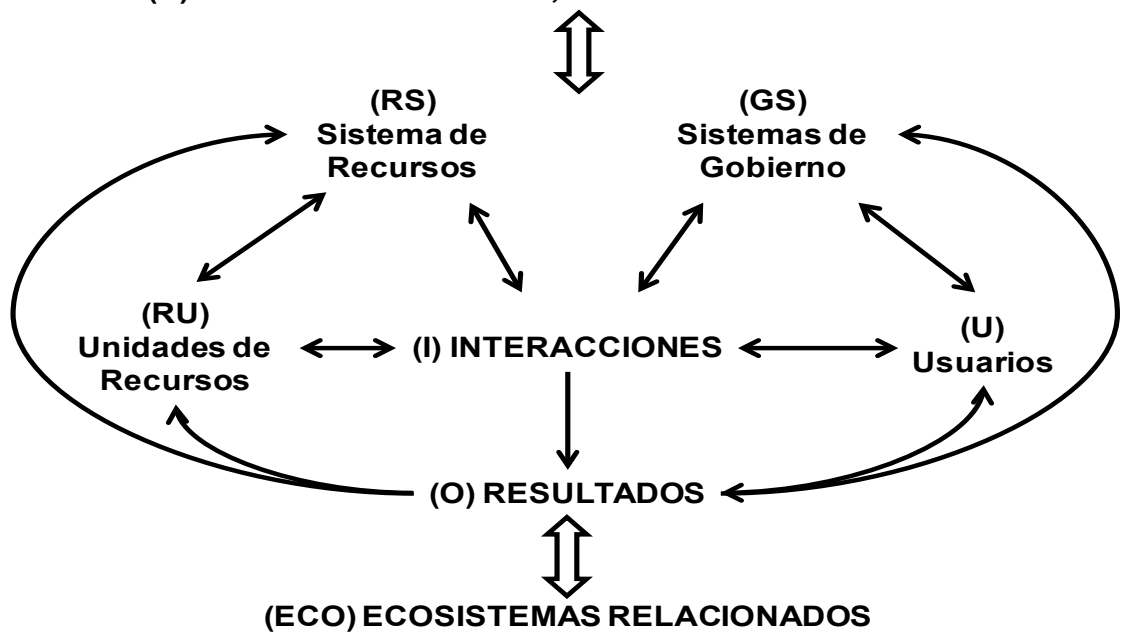

Fuente: Ostrom, 2009: 420

Los aportes de la teoría de los bienes comunes a la gestión comunitaria pueden leerse como una ruptura del paradigma de gobernanza sustentado en la dicotomía Estado - individuo.

En principio, la gobernanza de los bienes comunes destaca el valor de la acción colectiva y el rol de la comunidad como escenario para el ejercicio de los derechos civiles. La dicotomía entre los bienes públicos y los bienes privados hoy se cuestiona a partir de la teoría de los recursos de uso común o RUC, también llamados commons o bienes comunes (common-pool resources o CPR por sus siglas en inglés). Hess (2008: 32) explica la diferencia entre un bien común y un bien público, en los siguientes términos .

\footnotetext{
“(...) un bien común es un recurso compartido por un grupo donde el recurso es vulnerable al cercamiento, a la sobreexplotación y a los dilemas sociales. A
} 
diferencia de un bien público, este requiere administración y protección en orden a su sostenibilidad"

Boilier (2008: 38) anota además que

“(...) El meollo de llamar común a un recurso compartido, es destacar que el recurso pertenece a la gente, no al gobierno, y por lo tanto debe servir a propósitos más amplios que los que ofrece el mercado. Cuando se considera que un recurso es propiedad del gobierno, su vínculo legal y moral con la ciudadanía empieza a diluirse. Los bienes comunes ponen de relieve los derechos precedentes de los comuneros y de todo tipo de comunidades por encima del gobierno"

Estas reflexiones, reconocen en comunidad una identidad propia y los atributos para el ejercicio de los derechos de acceso a los recursos naturales, permitiendo la construcción de arreglos institucionales entre stakeholders.

En segundo lugar, la teoría de los bienes comunes aclara que los atributos de autorregulación y autogestión propios de las comunidades se manifiestan a través de las reglas constitutivas y las reglas de elección colectiva que son la expresión de los arreglos institucionales entre los diversos stakeholders, mientras que las reglas operacionales se desprenden de éstas, permitiendo la gestión cotidiana del recurso natural (Ostrom, 1990, citado en Berkes, 2009). Sin embargo, en la práctica es difícil establecer una división entre las reglas formales y su interpretación. Este debate sobre el pluralismo legal implícito en la gobernanza multinivel, alimenta la idea de que los modelos de gestión son un nivel de implementación de unas categorías funcionales (reglas constitucionales y reglas de elección colectiva) precedentes, en un nivel más operativo (reglas operacionales) sin perder su naturaleza normativa (Boelens y Zwarzteen, 2004).

En tercer lugar, la gobernanza de los bienes comunes implica también la gestión descentralizada. Andersson y Ostrom (2008) presentan un marco ampliado de gobernanza descentralizada y armonizada en diferentes niveles y con diferentes actores en un entorno participativo, involucrando nuevas instancias multinivel para facilitar la interacción entre los usuarios y el gobierno local y central, rol que cumplen unos niveles intermedios de gobernanza que pueden ser las asociaciones de usuarios y las ONG's (figura 2).

En cuarto lugar, la teoría de los RUC también recupera los valores colectivos (cooperación, altruismo, identidad) que reposan en la motivación para la acción colectiva más allá de la satisfacción del interés individual. A manera de ejemplo, en la construcción de pequeños abastos comunitarios 
Figura 2. Modelo conceptual de la gobernanza descentralizada desde una perspectiva policéntrica

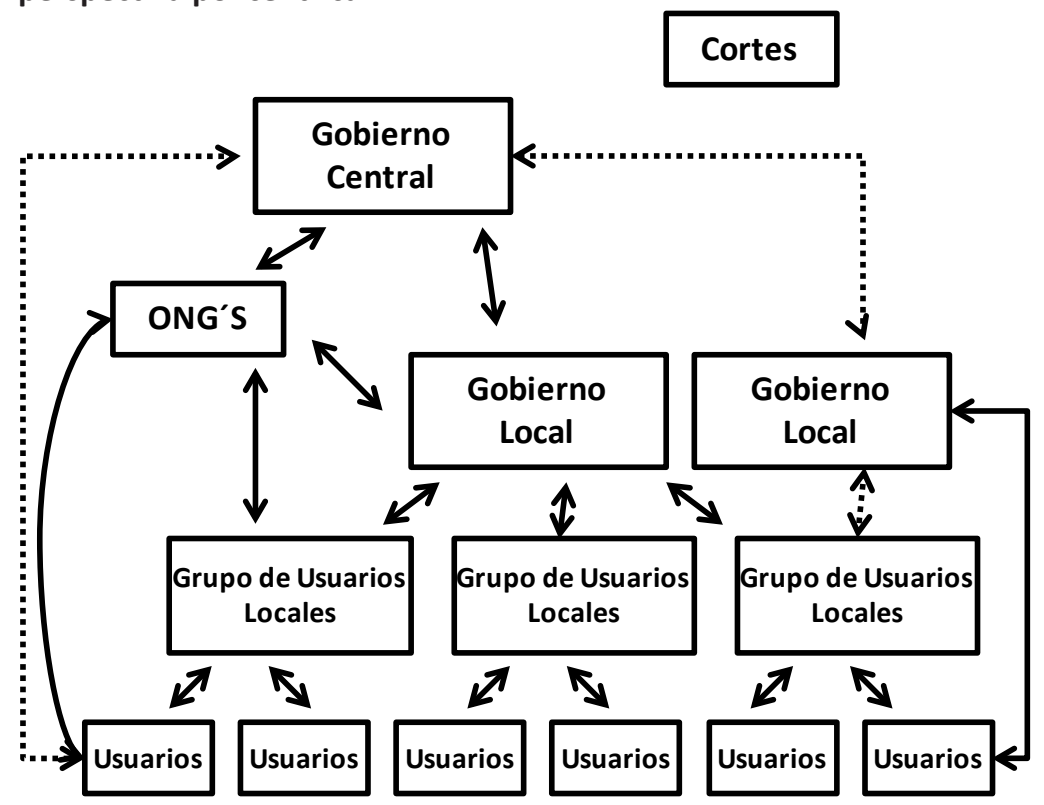

Fuente: Andersson y Ostrom, 2008: 73

de agua, aún es posible observar arreglos institucionales ancestrales como la minga o el convite, comunes en Colombia o Ecuador, que son jornadas de trabajo colectivo en la que toda la comunidad participa, sin que exista una compensación material por dicho trabajo. Los hombres excavan el paso de las tuberías, las mujeres preparan la comida y los niños ayudan con cargas livianas o llevando mensajes (Quintana, 2007). En estas actividades, puede percibirse cómo los bienes comunes hacen parte de los procesos de creación de valor en el seno de la comunidad y contribuyen a la satisfacción de las necesidades individuales y colectivas.

La gestión comunitaria vista desde la gestión colaborativa o co-gestión.

La evolución del concepto de gestión colaborativa o co-gestión, obedece a la necesidad de un modelo que permita la interacción eficiente de los actores o agentes encargados del manejo de un recurso determinado. El Banco Mundial (1999) definió inicialmente a la co-gestión como el compartir responsabilidades, derechos y deberes entre los actores primarios 
definidos como : (i) gobierno central; (ii) gobierno local; (iii) sector comercial y privado y; (iv) sociedad civil o comunidades locales. Posteriormente se identifica a la co-gestión como una estrategia de gobernabilidad de los recursos naturales en los siguientes términos:

La co-gestión puede ser entendida como una situación en la cual dos o más actores sociales negocian, definen y garantizan entre sí un reparto limpio y de las funciones de gestión, titularidad de derechos y responsabilidades para un territorio dado, área o grupo de recursos naturales (Borrini-Feyerabend et al., 2000: 1)

Esta definición es optimista pues a pesar de que la co-gestión se defina como un "reparto limpio" no siempre se llega a un reparto equitativo de poder. De hecho, la co-gestión puede desembocar en una negociación desfavorable para los más débiles en la que la toma de decisiones es impuesta por uno de los actores. Un modelo de co-gestión tampoco alcanza a establecer la titularidad de derechos, que usualmente responden a repartos de poder que van más alla de la gestión compartida de un determinado recurso. Por otra parte, la co-gestión se expresa a través de acuerdos o decisiones que pueden ir desde compartir información hasta establecer con detalle los repartos y la administración de un recurso determinado.

Carlsson y Berkes (2005) desarrollaron una imagen para describir las interacciones entre los agentes involucrados en la co-gestión, como se muestra en la figura 3:

Figura 3. Ejemplo de una red de co-gestión (Carlsson y Berkes 2005)

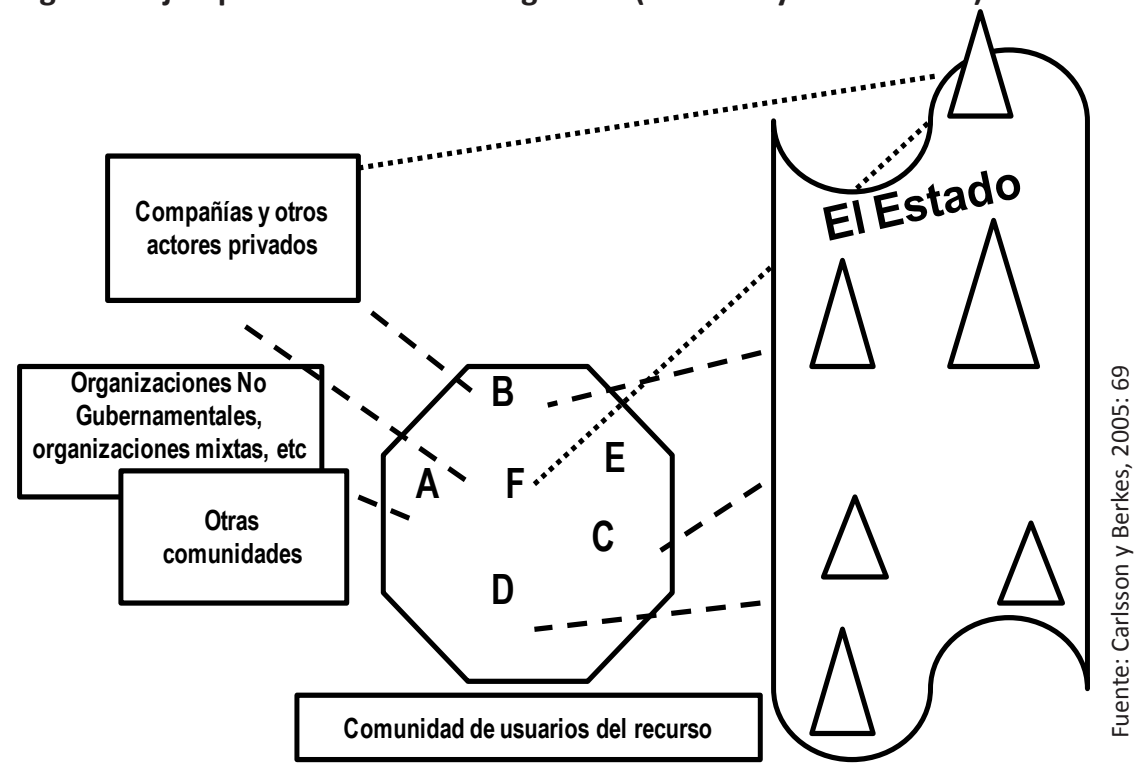


Este modelo representa los elementos esenciales de la co-gestión que surgen de su evolución conceptual: i) Diferentes niveles de gobierno; ii) diferentes actores involucrados, iii) diferentes relaciones establecidas entre los actores y los niveles de gobierno. Esto configura una estructura sistémica - dinámica a partir de las interacciones entre las funciones y los roles de los actores. Así los diferentes niveles de gobierno se explican por la representación de las jerarquías de poder en el Estado, que a su vez corresponden al ámbito de influencia-poder de cada uno de los actores, los diferentes actores están emparejados uno con otro (al involucrar al Estado, los usuarios locales del recurso, los actores comerciales, las organizaciones no gubernamentales y otro amplio rango de actores públicos y privados) y los problemas entre los diferentes niveles de gobierno y los diferentes actores se resuelven a través de alianzas (Carlsson y Berkes, 2005:69).

En todo caso, las complejas relaciones entre los actores no están exentas de dificultades (Leach, Mearns y Scoones, 1999 ; Eikeset et al, 2011). En primer lugar, los gobiernos nacionales y locales están conformados a su vez por múltiples agencias o dependencias con funciones reglamentadas, pero que se entrecruzan y confunden al ser aplicadas en la práctica. Por otra parte, bajo el poder-influencia de los representantes del Estado se configuran acuerdos o conexiones colaborativas que no necesariamente obedecen a una disposición legal. Por ejemplo, una norma nacional puede establecer los requerimientos mínimos para el control del agua potable, pero el control termina realizándose según los recursos y acuerdos presentes en la localidad. En segundo lugar, los actores privados (propietarios de tierras, de fuentes de financiamiento, de recursos y de medios de producción) pueden tener un interés individual (como la propiedad sobre un terreno) y simultáneamente un interés que comparte con la comunidad (como el abastecimiento de agua para su terreno) que pueden ser fuente de conflicto. En tercer lugar, el comportamiento de las comunidades frente a escenarios de toma de decisiones es altamente impredecible. Algunas comunidades eligen manifestarse como una sola voz a pesar de las diferencias de intereses al interior de la comunidad, mientras otras se caracterizan por la falta de cohesión y la exposición de sus diferencias internas (Carlsson y Berkes, 2005).

Como se observa, un modelo de co-gestión es más complejo que uno de gestión comunitaria, pues involucra un mayor número de actores y depende de factores exógenos que determinan la toma de decisiones para los acuerdos colaborativos (la legitimidad de los actores para intervenir en el sistema, los derechos de propiedad, los apoyos institucionales, (Berkes, 
2009). Para los recursos naturales, el mismo ecosistema interviene como un entorno impredecible y no lineal que altera las condiciones de gestión.

\section{Modelos de co-gestión aplicados al manejo del agua}

La revisión conceptual precedente establece los tres elementos esenciales de la co-gestión: i) los actores o stakeholders involucrados ; ii) las funciones o responsabilidades asignadas a los actores y ; iii) el territorio o recurso natural en el que se aplica esta estrategia. En esta estructura, los arreglos institucionales resultantes son el resultado de los equilibrios de poder alcanzados y las funciones deben ser operacionalizadas para el ejercicio de la gestión. Por otra parte, las debilidades de las comunidades para el abastecimiento de agua potable (Moriarty y Verdemato, 2010 ; Dobbin, 2013) y la necesidad de apoyo externo para garantizar la gestión apropiada del vital recurso (Lockwood, 2002) invitan a la exploración de la co-gestión como estrategia de política pública para el acceso al agua y al saneamiento, dado su énfasis en la participación de actores clave desde diferentes niveles de gobierno y desde distintos sectores de la sociedad. A continuación se presentan los modelos de co-gestión documentados en la literatura, descritos en términos de su estructura y sus funciones asociadas.

Modelo del Desarrollo Conducido por la Localidad y la Comunidad (LCDD)

Inicialmente, la gestión comunitaria, también conocida como Community Based Management (CBM por sus siglas en inglés) fue estudiada como alternativa de gestión autónoma, identificándose como "Desarrollo Conducido por la Comunidad" o

Community Driven Development (CDD por sus siglas en inglés) (Lockwood, 2002). Posteriormente, Binswanger-Mkhize y De Regt (2010) presentan la estrategia de "Desarrollo Conducido por la Localidad y la Comunidad" o Local Community Driven Development (LCDD por sus siglas en inglés), bajo una estructura tripartita en la que la co-gestión se asume en conjunto por los stakeholders sectoriales, por el gobierno descentralizado y por la comunidad (figura 4). 


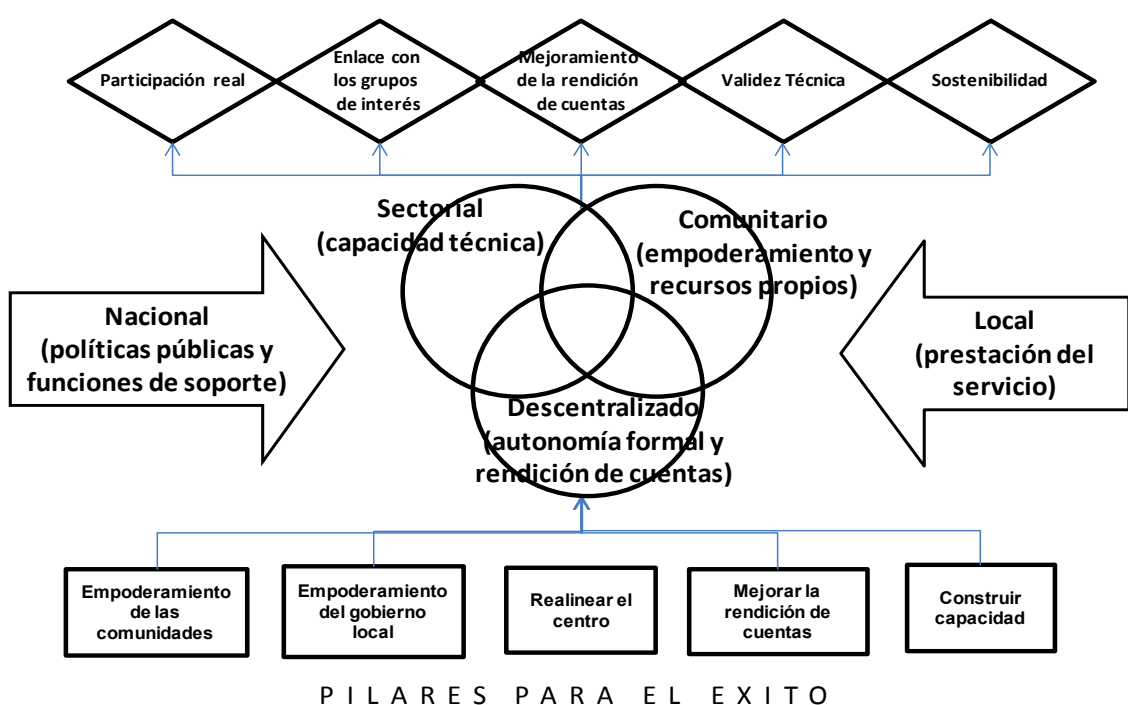

Fuente: Binswanger-Mkhize et. al. 2010: 6. Traducción y figura de Bernal (2011).

El modelo LCDD responde a las necesidades de concertación y coordinación de las acciones del estado y de los particulares sobre los recursos naturales en el ámbito local, y facilita el desarrollo de modelos de gestión con un enfoque territorial y funcional, bajo las premisas del empoderamiento de las comunidades, el empoderamiento del gobierno local, la descentralización (realinear el centro), la rendición de cuentas y la construcción de capacidad.

De hecho el estudio y la implementación de modelos de co-gestión para el manejo del agua están apoyados por el Programa de Seguridad del Agua (Water Safety Program) bajo diferentes iniciativas de alcance local, regional y nacional como son el "Proyecto para el Mejoramiento Global del Saneamiento" (figura 5) en India, Indonesia y Tanzania, y en el Modelo de Saneamiento Básico integral SABA (figura 6), adelantado en ocho regiones de Perú. Estos proyectos de alcance nacional han demostrado un impacto positivo en los indicadores de abastecimiento de agua en la última década en los países de su implementación según múltiples publicaciones (Water Safety Program, 2013) pero su eficiencia como modelo de gestión y como alternativa de gobernanza en el largo plazo aún está por evaluarse, pues 
los proyectos mencionados aún siguen siendo acompañados por un agente externo (ONG o agencias de cooperación) y los Estados beneficiarios aún no ofrecen estabilidad institucional y económica suficiente para garantizar la sostenibilidad de estas estrategias.

Figura 5. El rol y las funciones del gobierno local en el Proyecto para el Mejoramiento Global del Saneamiento

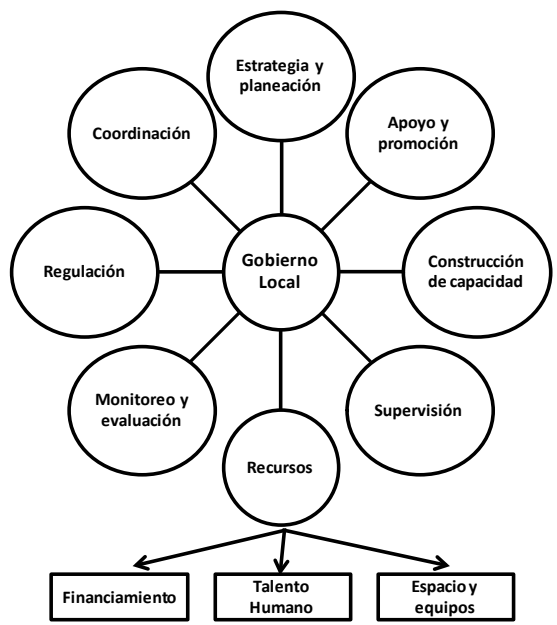

Fuente: Adaptado de Rosensweig y Kopitopoulos, 2010: 4

Figura 6. Modelo conceptual para la Gestión de Servicios de Saneamiento PROPILAS -SABA

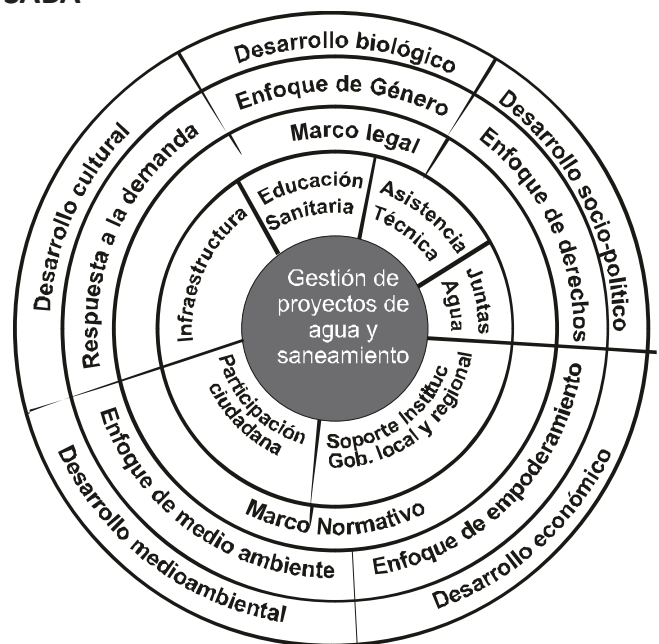

Fuente: COSUDE, CARE, WSP y Gob. Regional de Cajamarca, 2011: 12. 
La propuesta "Triple S" (Lockwood y Smits, 2011) indica que la prestación de servicios de agua y saneamiento mediante la gestión comunitaria requiere de la creación de un ambiente propicio que facilite la asignación de roles y funciones de cada uno de los niveles de gobierno frente al abastecimiento del agua. Para ello propone los "Modelos Consensuados de Servicios de Suministro" (Sustainable Services at Scale o Triple S) que son acuerdos entre diferentes niveles de gobierno para la prestación del servicio bajo un enfoque de suministro del servicio a los usuarios siendo la infraestructura y los sistemas medios para proporcionarlo.

Una propuesta nacional construida a la manera de Triple $S$ se debe ajustar por las políticas existentes de un país y por el marco legal que define: i) normas y estándares para el suministro rural del agua; ii) roles, derechos y responsabilidades; y iii) mecanismos de financiamiento. También asigna roles a cada uno de los niveles de gobernanza o niveles de descentralización (figura 7):

Figura 7. Modelo para la entrega del servicio de Lockwood y Smits, 2011

\begin{tabular}{|c|c|c|c|c|}
\hline \multicolumn{4}{|c|}{$\begin{array}{l}\text { Ambiente propicio: políticas, marcos institucionales y legales, planeación de } \\
\text { inversiones de gran escala, aprendizaje e innovación }\end{array}$} & $\begin{array}{l}\text { Nivel nacional o } \\
\text { estadual }\end{array}$ \\
\hline \multicolumn{4}{|c|}{$\begin{array}{l}\text { Funciones de la autoridad de servicios: planeación, contratación, monitoreo, } \\
\text { apoyo posconstrucción y aprendizaje }\end{array}$} & Gobierno Local \\
\hline \multicolumn{4}{|c|}{$\begin{array}{l}\text { Proveedores de servicios: operaciones cotidianas, administración y } \\
\text { mantenimiento }\end{array}$} & \multirow{2}{*}{$\begin{array}{c}\text { Nivel comunitario o } \\
\text { del sistema de } \\
\text { abastecimiento }\end{array}$} \\
\hline $\begin{array}{l}\text { Gestión } \\
\text { comunitaria }\end{array}$ & $\begin{array}{l}\text { Operadores del } \\
\text { sector público }\end{array}$ & $\begin{array}{l}\text { Operadores del } \\
\text { sector privado }\end{array}$ & $\begin{array}{l}\text { Auto- } \\
\text { abastecimiento }\end{array}$ & \\
\hline $\begin{array}{l}\text { Modelo de } \\
\text { entrega del } \\
\text { servicio }\end{array}$ & $\begin{array}{l}\text { Modelo de } \\
\text { entrega del } \\
\text { servicio }\end{array}$ & $\begin{array}{l}\text { Modelo de } \\
\text { entrega del } \\
\text { servicio }\end{array}$ & $\begin{array}{l}\text { Modelo de } \\
\text { entrega del } \\
\text { servicio }\end{array}$ & \\
\hline
\end{tabular}

Fuente: Lockwood y Smits, 2011: 22. Traducción de los autores.

\section{Análisis comparado de los modelos de co-gestión aplicados al abastecimiento de agua potable}

Los modelos presentados coinciden al señalar que el desarrollo de un modelo nacional, regional o local depende del marco legal y de las políticas públicas que definen las condiciones de prestación del servicio, enfati- 
zando en la necesidad de multiples niveles de gobierno. A partir de estas consideraciones, cada modelo presenta una estructura que determina la posición de los diferentes actores o stakeholders en el escenario local, frente a diferentes problemáticas ambientales, sociales y económicas.

La definición de las funciones necesarias para el adecuado abastecimiento de agua depende del enfoque operacional de cada una de las estrategias propuestas. Mientras algunos modelos ponen el acento en el aseguramiento de las condiciones materiales para la prestación del servicio - recursos financieros, capacitación, información, tecnologías - (Lockwood y Smits, 2011) otros conceden un mayor despliegue a las fortalezas organizacionales - coordinación, estructuras de apoyo (Rosensweig y Kopitopoulos, 2010). También existen esfuerzos enfocados al fortalecimiento de capacidades que se concentran en una función específica y en la estructura necesaria para su despliegue exitoso.

Sin embargo, la enumeración de más o menos funciones o la disposición de una estructura de gobierno, no garantizan la efectividad de un modelo de co-gestión. De hecho, la desarticulación orgánica y funcional entre los actores es la causa principal del pobre aprovechamiento de los recursos disponibles (Fonseca y Bolt, 2002). Es por ello que resulta pertinente definir una estrategia de intervención a partir de la identificación de las funciones fundamentales para el abastecimiento de agua a las comunidades asignadas al nivel de gobierno mas conveniente, para luego asignar dichas funciones a los actores que tengan mayor vocación para contribuir a la autorregulación y autogestión de las comunidades y así desarrollar alternativas de co-gestión sostenibles.

Modelo de co-gestión para los pequeños abastecimientos comunitarios de agua en Colombia

Colombia es un estado coordinado desde el gobierno central, cuyas funciones descentralizadas se delegan en los 32 departamentos y 1,112 municipios. Los departamentos que son entes regionales que agrupan a varios municipios pero que no tienen autonomía regulatoría, cumplían ciertas funciones de asignación del gasto en infraestructura a través de los planes departamentales de agua hasta 2012 y de control de la calidad del agua, mientras que el municipio continúa siendo el garante del acceso al agua y al saneamiento, en las áreas urbanas y rurales de su jurisdicción. Si bien existe una norma nacional para regular los servicios públicos domiciliarios, entre ellos el agua potable, esta norma se aplica indistintamente a 
todos los operadores. No existe aún una regulación especial para el abastecimiento de agua y saneamiento en áreas rurales, pero el actual Plan Nacional de Desarrollo de Colombia (2010-2014) obliga a la definición de los lineamientos para ello. En este contexto, se presenta el modelo de cogestión Bernal-Rivas (ver figura 8) que puede ser útil como orientación para el desarrollo normativo y de políticas públicas de apoyo a la gestión comunitaria del agua.

Figura 8. Modelo Bernal - Rivas para la articulación funcional de la co-gestión

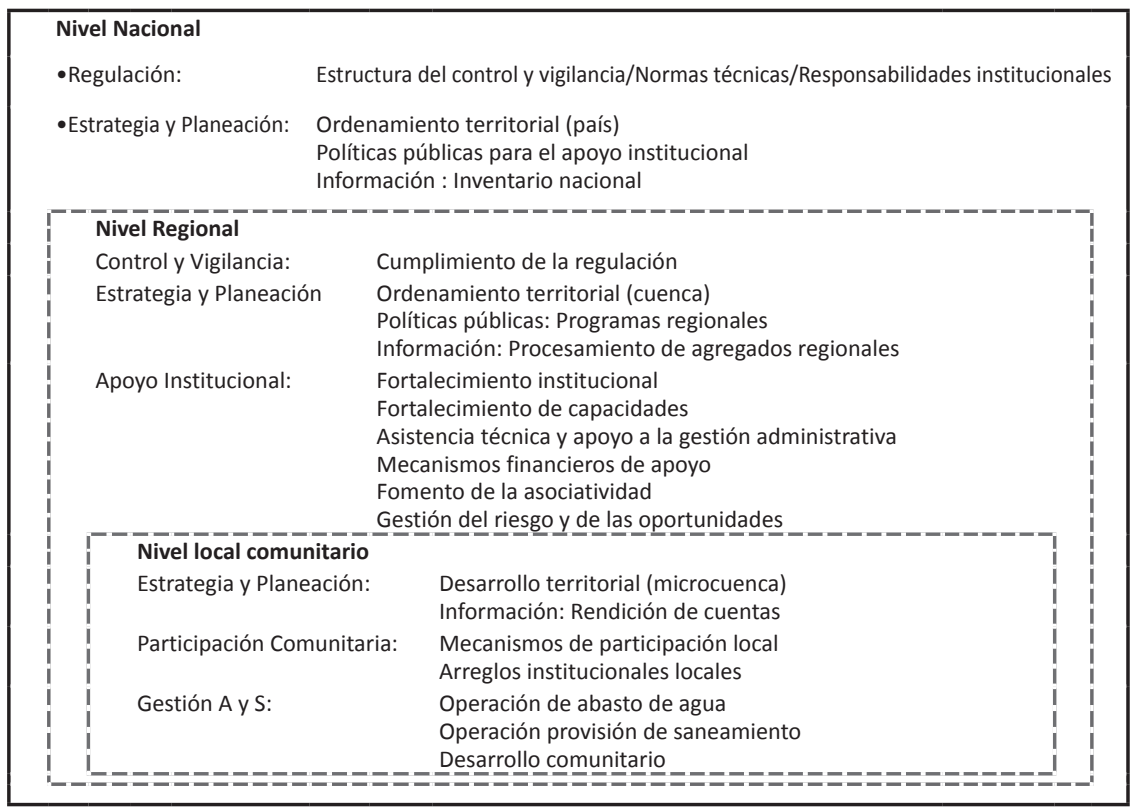

Fuente: Elaboración de los autores

Si bien para algunos investigadores sólo se puede llamar modelo a aquella propuesta que esté sustentada en evidencia empírica comprobable y replicable, un modelo también puede ser de tipo conceptual. El propósito de un modelo conceptual (Rivas, 2013) es el de establecer una estructura para la comprensión de una realidad compleja En tal sentido, el modelo Bernal - Rivas es de tipo conceptual, estructurado en diferentes niveles de gobierno a la manera de Lockwood y Smits (2011) pero presentados como espacios anidados. El modelo considera las funciones esenciales para el abastecimiento de agua potable presentadas por Rosensweig y Kopitopoulos (2010), Lockwood y Smits (2011) y el modelo SABA e incluye otras funciones que surgen de la experiencia en gestión del agua pero que 
no fueron claramente expuestas en dichos modelos (como la asociatividad y gestión del riesgo).

El modelo Bernal-Rivas pretende responder a las necesidades crecientes de articulación de las funciones de los stakeholders en la provisión de agua apta para consumo humano, propicia la gestión de los recursos naturales o del territorio desde el nivel local, uniendo a la comunidad, al gobierno (nacional o local), al sector privado y a la sociedad civil y estimula la participación de niveles intermedios de gobierno o de organizaciones transitorias (como las agencias de cooperación o las ONG's, o las asociaciones de segundo nivel) para las actividades de apoyo a la gestión comunitaria

Los arreglos institucionales necesarios para las interacciones entre los niveles de gobierno, según se formulan en la figura 8 , se sustentan en la posibilidad de la acción coordinada y cooperante entre los diferentes niveles del gobierno colombiano y los actores intermedios y las comunidades. Las relaciones verticales de coordinación se producen cuando una instancia de gobierno superior tiene la potestad de ordenar o condicionar las decisiones de una instancia inferior. Esto es muy común en los aspectos regulados por el Estado bajo un criterio jurídico formal que se impone en virtud de la autoridad. En tal medida, las relaciones de coordinación son reglas constitutivas del modelo. Simultáneamente, pueden surgir relaciones horizontales de cooperación, cuando diferentes instancias de gobierno deciden actuar de manera conjunta y armónica para el logro de un propósito común, a través de reglas de elección colectiva y reglas operativas que permiten el desarrollo de la autorregulación y la autogestión. Las reglas en todos los niveles deberían ser fruto de un consenso entre los diferentes niveles de gobierno, asignando el presupuesto, el talento humano y los espacios de concertación necesarios para la participación comunitaria.

La estructura del modelo se ajusta al contexto jurídico de Colombia como Estado Unitario territorial en tres niveles: i) nacional; ii) regional o departamental; y iii) local-municipal/comunitario. 
El nivel nacional tiene plena autonomía regulatoria y está a cargo del diseño de políticas públicas de alcance nacional. Por ello en este nivel reposan las funciones normativas y de diseño estratégico, como son las normas técnicas y los procedimientos y sanciones para el control y vigilancia, las estructuras, procesos y procedimientos preestablecidos para la gestión o manejo de los recursos hídricos, los lineamientos para alcanzar los indicadores de desarrollo como agregados nacionales y la ejecución del gasto correspondiente (cuadro 1).

\section{Cuadro 1. Modelo Bernal - Rivas. Funciones del nivel nacional}

\begin{tabular}{|l|l|}
\hline Regulación & $\begin{array}{l}\text { Normas técnicas: (calidad del agua, calidad del servicio, modelos tarifarios, requisitos } \\
\text { formales para la operación del agua). } \\
\text { Mecanismos de control y vigilancia: procedimientos, sanciones, y requerimientos para la } \\
\text { rendición de cuentas. } \\
\text { Responsabilidades institucionales: metodologías, manuales y protocolos. }\end{array}$ \\
\hline $\begin{array}{l}\text { Estrategia } \\
\text { planeación }\end{array} \quad \boldsymbol{y}$ & $\begin{array}{l}\text { Ordenamiento territorial del Estado: genera las pautas para el ordenamiento territorial } \\
\text { regional y el desarrollo territorial local y comunitario (por ejemplo, la definición de áreas } \\
\text { naturales protegidas, o los lineamientos generales para la protección de los acuíferos). } \\
\text { Politicas públicas para el apoyo institucional : Investigaciones de nivel nacional, asesoría } \\
\text { experta y programas para el desarrollo de capacidades y proyectos para favorecer la gestión } \\
\text { de conocimiento, coordinadas con otras estrategias nacionales formuladas para la superación } \\
\text { de la pobreza y el desarrollo local. } \\
\text { Información: Establecer mecanismos de reporte de información que permitan contar con } \\
\text { inventarios nacionales e indicadores agregados para la toma de decisiones. }\end{array}$ \\
\hline
\end{tabular}

Fuente: Elaboración de los autores

\section{Nivel Regional}

El nivel regional, que en Colombia corresponde al departamento y reúne a los municipios próximos, permite incorporar ciertas funciones de enfoque territorial para el agua y saneamiento que son beneficiadas por criterios de escala o diversidad, permitiendo su manejo en contextos ambientales, sociales y culturales homogéneos. En este nivel es posible articular la conexión entre el nivel nacional y comunitario mediante tareas compartidas y complementarias con una mayor participación comunitaria directa (cuadro 2). 


\begin{tabular}{|c|c|}
\hline $\begin{array}{l}\text { Control } \\
\text { Vigilancia }\end{array}$ & $\begin{array}{l}\text { Unidades regionales para el control y vigilancia que presten una atención efectiva y cercana a } \\
\text { los problemas que puedan derivarse de la vulneración de los estándares mínimos de calidad, } \\
\text { continuidad y acceso al agua }\end{array}$ \\
\hline $\begin{array}{l}\text { Estrategia } y \\
\text { planeación }\end{array}$ & $\begin{array}{l}\text { Ordenamiento territorial a nivel de cuenca: la planeación regional permite estructurar } \\
\text { respuestas más oportunas frente a los riesgos derivados del cambio climático, de los } \\
\text { regímenes de lluvias y de la contaminación. } \\
\text { Politicas públicas para el desarrollo de programas y proyectos: En la escala regional es } \\
\text { posible contar con condiciones homogéneas y ventajas de economías de escala } \\
\text { Información: procesamiento de agregados regionales: para alimentar los resultados de los } \\
\text { programas y proyectos adelantados y entregar información importante para la estrategia y la } \\
\text { planeación en el nivel regional y nacional. }\end{array}$ \\
\hline $\begin{array}{l}\text { Apoyo } a \\
\text { la gestión } \\
\text { comunitaria: }\end{array}$ & $\begin{array}{l}\text { Fortalecimiento institucional: mejoramiento del desempeño del gobierno local (municipio) reconocimiento y } \\
\text { constitución formal de los operadores y asignación de funciones y recursos. } \\
\text { Fortalecimiento de capacidades: mejoramiento de los conocimientos y habilidades de gestión en la } \\
\text { comunidad. Mejora de la participación comunitaria, del desempeño operativo y administrativo o difusión } \\
\text { de hábitos higiénicos. } \\
\text { Asistencia técnica y apoyo a la gestión administrativa: acompañamiento permanente o eventual a las } \\
\text { comunidades (provisión de insumos y equipos, apoyo en las reparaciones, asesoría técnica y administrativa } \\
\text { y apoyo en los procesos de rendición de cuentas) } \\
\text { Fomento a la asociatividad: espacios para la cohesión entre comunidades ayudando a mejorar la } \\
\text { gobernabilidad local, generar economías de escala y desarrollar capital social. } \\
\text { Gestión del riesgo y de las oportunidades: apoyo a las comunidades en su respuesta frente a los riesgos y las } \\
\text { oportunidades. (como articular acciones colectivas ante la presencia de eventos de riesgo o catastróficos, o } \\
\text { aprovechar los eventos sociales y culturales de la región para promover el cuidado del agua).. }\end{array}$ \\
\hline
\end{tabular}

Fuente: Elaboración de los autores

\section{Nivel local/comunitario}

El nivel local/comunitario en Colombia está integrado por la acción conjunta entre el municipio y las comunidades rurales. Si bien el municipio ostenta total control en el abastecimiento de agua en el casco urbano, en las áreas rurales se requiere una cooperación estrecha y cotidiana entre el municipio y las comunidades siendo difícil distinguir cuales son las tareas y responsabilidades de una y otra institución, más aún cuando estos arreglos se ciñen a reglas de elección colectiva y operativas que surgen de la entre las comunidades encargadas del abastecimiento de A y S, las autoridades y los vecinos del municipio, en una dinámica de cooperación y concertación. Por otra parte, la gestión comunitaria del agua y el saneamiento (desde una perspectiva de manejo integral del recurso hídrico) requiere un alto desempeño en las operaciones cotidianas de los sistemas de abastecimiento. Las comunidades rurales están en permanente contacto directo con el recurso natural, sin embargo, estas funciones requieren de un permanente acompañamiento desde el nivel local (municipio), apoyo 
desde el nivel local y una estructura constitutiva fijada desde el nivel nacional (cuadro 3).

\section{Cuadro 3. Modelo Bernal - Rivas. Funciones del nivel local/comunitario}

\begin{tabular}{|c|c|}
\hline $\begin{array}{l}\text { Estrategia } \\
\text { planeación }\end{array}$ & $\begin{array}{l}\text { Participación en el desarrollo territorial de la microcuenca: Es el escenario propicio para } \\
\text { fomentar las relaciones entre comunidades aguas arriba, que habitan cerca de las fuentes de } \\
\text { agua y aguas abajo, que reciben los efluentes de los centros urbanos cercanos. La planeación } \\
\text { concertada mejora la dinámica urbano-rural y fomenta el desarrollo local. } \\
\text { Alimentación de sistemas de información: Es necesario que el municipio y las comunidades } \\
\text { cooperen en la rendición de cuentas según parámetros de reporte de datos, generación } \\
\text { de indicadores y evaluación del desempeño acordes a las capacidades de las comunidades } \\
\text { campesinas y a las necesidades de la gestión municipal }\end{array}$ \\
\hline $\begin{array}{l}\text { Participación } \\
\text { comunitaria: }\end{array}$ & $\begin{array}{l}\text { Mecanismos de participación local: Convocatoria a asambleas, conformación de los estatutos, } \\
\text { elecciones colectivas, respeto a las condiciones de gobernanza participación en asociaciones } \\
\text { de segundo nivel, información oportuna sobre circunstancias de riesgo. } \\
\text { Arreglos institucionales locales: Reconocimiento de la comunidad como actor colectivo } \\
\text { que interactúa con otros actores de los diferentes niveles de gobierno y promoción de las } \\
\text { relaciones en el nivel local y comunitario (como la participación en asociaciones de segundo } \\
\text { nivel, gestión de acuerdos con el gobierno municipal).. }\end{array}$ \\
\hline $\begin{array}{l}\text { Gestión del } \\
\text { agua y el } \\
\text { saneamiento: }\end{array}$ & $\begin{array}{l}\text { Operación de abasto de agua: manejo cotidiano de los equipos o instalaciones asociados al aprovechamiento } \\
\text { del recurso } \\
\text { Operación de Saneamiento: Desde una visión de la gestión integrada del recurso hídrico, la provisión de } \\
\text { agua implica la solución de saneamiento. Si bien algunas veces en las áreas rurales no es viable contar con } \\
\text { sistemas de alcantarillado en red, la comunidad puede hacer mucho para contribuir a la construcción y } \\
\text { mantenimiento de alternativas individuales de saneamiento. } \\
\text { Desarrollo Comunitario: Aplicación de las orientaciones de gestión para la toma de decisiones en operaciones } \\
\text { cotidianas, el archivo de documentos, la rendición de cuentas y la solución de controversias y observancia de } \\
\text { las reglas internas de la comunidad (estatutos, procesos de toma de decisiones). }\end{array}$ \\
\hline
\end{tabular}

Fuente: Elaboración de los autores

\section{Conclusiones}

En Colombia, cerca de 12,000 organizaciones comunitarias abastecen de agua aproximadamente a siete millones de personas y son una forma autorizada para la prestación de los servicios básicos de agua y saneamiento. Este trabajo busca proponer un modelo de co-gestión que contribuya al suministro de agua potable, teniendo en cuenta que la gestión comunitaria ayuda a los colectivos marginados que históricamente en Latinoamérica han carecido de voz para su acceso efectivo al agua y simultáneamente les permite adquirir experiencia en la gestión de otros recursos naturales a partir de la acción colectiva, lo que fortalece las estructuras sociales subyacentes para el desarrollo local.

También es posible afirmar que la gestión comunitaria responde a un contexto más complejo que la división entre la gestión pública y a la ges- 
tión privada, y en tal sentido configura una ruptura de paradigmas bajo la teoría de los recursos de uso común (RUC). Sin embargo, aún falta un largo camino por recorrer en la comprensión de la gestión comunitaria desde la perspectiva de la gobernanza y la motivación para la acción colectiva en el seno de la comunidad. Lo anterior resulta de suma importancia puesto que la gestión de los recursos naturales está aún lejos de producirse en condiciones de equidad, eficiencia y respeto por el equilibrio ambiental.

Si bien existen diferencias entre las aproximaciones teóricas a la gestión comunitaria- capital social, teoría de los bienes comunes y la co-gestión-, estas tres vertientes forman parte de un corpus intelectual complementario de vanguardia en las ciencias sociales contemporáneas para el desarrollo del tejido social, la cooperación y el empoderamiento comunitario. Por otra parte, la gestión comunitaria está en el origen de los arreglos institucionales para el manejo de los recursos naturales, pero la co-gestión permite el involucramiento de un numero plural de actores desde diferentes sectores, necesarios para los consensos duraderos y el compromiso institucional requerido para el abastecimiento de agua potable. En contraste con la imagen ideal de una organización formal y jerarquizada, la co-gestión es un proceso en el cual las partes, su poder y relativa influencia, posiciones $\mathrm{y}$ actividades son continuamente reajustadas, y por ello las estrategias de co-gestión implementadas para el abastecimiento de agua responden a diferentes estructuras y funciones que interactúan en un sistema dinámico en permanente cambio y evolución, como resultado de los subsistemas que lo integran. Así, a partir de los fundamentos teóricos y empíricos que sustentan a la gestión comunitaria, es posible diseñar modelos que orienten las decisiones de la administración pública en orden a la provisión eficiente de agua para las comunidades rurales. Las teorías de los bienes comunes y el capital social son enfoques enriquecedores de una propuesta de cogestión, por resaltar el potencial de la comunidad como creadora de valor y como escenario para la cooperación y la acción colectiva y por lo tanto pueden conducir a un modelo de co-gestión centrado en la comunidad como eje del desarrollo local. 


\section{Bibliografía}

Andersson, Krister y Elinor Ostrom, 2008, “Analyzing decentralized resource regimes from a polycentric perspective", Policy Sci, vol. 41, pp. 71-93.

AVINA, 2011, Modelos de Gobernabilidad Democrática para el Acceso al Agua en América Latina, http://avina.net/eng/wp-content/uploads/2011/09/697.pdf

Awortwi, Nicholas, 2012, "The riddle of community development: factors influencing participation and management in twenty-nine African and Latin American communities", Community Development Journal, vol. 48, núm. 1, pp. 89-104.

Berkes, Fikret, 2009, “Evolution of co-management: Role of knowledge generation, bridging organizations and social learning", Journal of Environmental Management, vol. 90, pp. 1692-1702.

Bernal, Andrea, 2011, "Hacia un Modelo de Co-gestión para OCSAS en Colombia”, en Pérez, Mario, J. Rojas, y R. Galvis, (eds.), Sociedad y Servicios Ecosistémicos, Cali, Universidad del Valle, pp. 557-568.

Binswanger-Mkhize, H., J. De Regt y S. Spector, (eds.), 2010, Local and Community Driven Development: Moving to Scale in Theory and Practice, World Bank Publications.

Binswanger-Mkhize, H., J. De Regt y S. Spector, (eds.), 2009, Scaling Up Local E Community Driven Development (LCDD). A Real World Guide to Its Theory and Practice, World Bank Publications.

Boelens, Rutgerd y Marguerite Zwarteen, 2004, "Pluralismo legal, derechos locales y gestión del agua. Entre el reconocimiento analítico y la estrategia política", en Francisco Peña (ed.), Los pueblos indígenas y el agua: desafíos del siglo XXI, Bogotá, Editorial Talleres Panamericana.

Boelens, Rutgerd, 2002, Derechos de agua, gestión indígena y legislación nacional, Universidad de Wageningen. http://www.bvsde.paho.org/bvsacd/cd29/lucha. pdf

Boilier, David, 2008, "Los bienes comunes: Un sector soslayado de la creación de riqueza”, en Silke Helfrich (comp.), Genes, Bytes y Emisiones : Bienes Comunes y Ciudadanía, México, Editorial H. Boll, pp. 30 - 41 
Borrini-Feyerabend, G., M.T., Farvar, J.C., Nguinguiriy y V. Ndangang, 2000, Comanagement of Natural Resources: Organising, Negotiating and Learning-by-Doing, Heidelberg, GTZ and IUCN, Kasparek Verlag. Reimpresión 2007.

Bourdieu, Pierre, 1986, "The Forms of Capital", en J. G. Richardson, Handbook of Theory and Research for the Sociology of Capital, New York, Greenwood Press.

Carlsson, Lars y F. Berkes, 2005, “Co-management: concepts and methodological implications", Journal of Environmental Management, vol. 75, pp. 65-76

Dobbin, Kristin, 2013, Comanagement and the fight for Rural Water Justice. Learning from Costa Rica, ASADAS. Prizer Senior Thesis, Paper 33, http://scholarship. claremont.edu/pitzer_theses/33

Eikeset, Anne, A. Ritcher, F. Diekert, D. Dankel, y N. Stenseth, 2011, “Unintended consequences sneak in the back door: making wise use of regulations in fisheries management", en A. Belgrano y Charles Fowler (eds.), Ecosystem Based Management for Marine Fisheries: An Evolving Perspective, Cambridge, Cambridge University Press, pp. 183 -217.

Etzioni, Amitai, 1993, El espíritu de la comunidad, Bogotá, Norma.

Feldman, Tine y Susan Assaf, 1999, Social Capital. Conceptual Frameworks and Empirical Evidence, New York: World Bank.

Fortuin, Karen, A. Van Koppen, y R. Leemans, 2011, “The value of conceptual models in coping with complexity and interdisciplinarity in environmental sciences education", Bioscience, vol. 61, núm. 10, pp. 802-814.

Fonseca, Catarina, y Evelyn Bolt, 2004, Cómo apoyar la gestión comuntaria de los sistemas de abastecimiento de agua. Guía para administradores, Delft. Países Bajos IPC. Centro Internacional de Agua y Saneamiento (Documentos Técnicos - Serie 37).

Grootaert, Christiaan, 1998, "Social Capital: the missing link?", en Social Capital Initiative, Working Paper No. 3, Washington, D.C., World Bank.

Helfrich, Silke, 2008, “Commons, ámbitos o bienes comunes, procomún o "lo nuestro" Complejidades de la traducción de un concepto", en Silke Helfrich (comp.), Genes, Bytes y Emisiones : Bienes Comunes y Ciudadanía, México, Editorial H. Boll, pp. 42- 48 . 
Hess, Charlotte, 2008, "Mapping the new commons", ponencia presentada en la 12th Biennial Conference of the International Association for the Study of the Commons, Universidad de Gloucestershire.

Leach, Melissa, Robin Mearnsy y Ian Scoones, 1999, “Environmental Entitlements: Dynamics and Institutions in Community-Based Natural Resource Management", World Development, vol.27, pp. 225-247.

Lockwood, Harold y Stef Smits, 2011, Supporting rural water supply, IRC International Water and Sanitation Centre and Aguaconsult.

Lockwood, Harold, 2002, Mecanismos de apoyo institucional para los sistemas rurales de agua potable y saneamiento manejados por las comunidades en América Latina, Environmental Health Project (EHP), Washington, D.C. http://www. ehproject.org/PDF/Strategic_papers/EHP\%20SR-6SPANISH.pdf

Moriarty, Patrick y Verdemato, Tania, 2010, Report of the International Symposium on Rural Water Services. Providing Sustainable Water Services at Scale, 13 15 April 2010. Kampala, Uganda. http://www.aguaconsult.co.uk/uploads/ pdfs/Report\%20Symposium\%20on\%20ural\%20Water\%20Services.pdf

Narayan, Deepa y Ian Pritchett, 1997. “Cents and Sociability: Household Income and Social Capital in Rural Tanzania", Policy Research Working Paper 1796, Washington, D.C., World Bank.

Ostrom, Elinor, 2009, “A General Framework for Analyzing Sustainability of SocialEcological Systems”, Science, vol. 325, núm. 5939, pp. 419-422.

Ostrom, Elinor, 1990, El gobierno de los bienes comunes. La evolución de las instituciones de acción colectiva, México, FCE, UNAM, IIS. 2a . Edición publicada en español en 2011.

Palerm, Jacinta y Tomás Martínez, (eds.), 2009, Aventuras con el agua. La Administración del agua de riego, historia y teoría, México, Colegio de Posgraduados.

Putnam, Robert, 1993, Making Democracy Work. Civic traditions in modern Italy, Princeton, NJ, Princeton University Press.

Quintana, Ana, 2008, El conflicto por la gestión del servicio de acueducto en Dosquebradas (Risaralda - Colombia) Un estudio desde la ecología política. Tesis de Doctorado. Barcelona: Universidad de Barcelona. 
Rapoport, Anatole, y A.M. Chammah, 1965, Prisoner's Dilemma: A study in Conflict and Cooperation, Michigan, University of Michigan Press.

Rivas, Luis, 2013, ¿Comó hacer una tesis de maestría?, México, Trillas.

Rojas, Johnny, P. Tamayo y M. García, 2011, Abastecimiento de Agua en Zonas Rurales. Colombia. Experiencias en la prestación de servicios sostenibles, La Haya, Centro Internacional de Agua Potable y Saneamiento (IRC), www.waterservicesthatlast. org/.../Colombia\%20country\%20study.pdf

Rosensweig, Fred, y Derko Kopitopoulos, 2010, Building the Capacity of Local Government to Scale Up Community-Led Total Sanitation and Sanitation Marketing in Rural Areas, Water Safety Program, Washington, D.C., World Bank.

Water and Sanitation Program (WSP), 2013, Scaling Up Rural Sanitation: Publications and Tools, http://www.wsp.org/global-initiatives/publications-and-tools0\#learning

WHO \& UNICEF, 2012, Progress on Drinking Water and Sanitation: 2012 Update.

Woolcock, Michael, 1998, "Social Capital and Economic development: Toward a Theoretical Synthesis and Policy Framework", Theory and Society, núm. 27, pp. 151-208

World Bank, 1999, Report from the International Workshop on Community-Based natural Resource Management, Washington, D.C., 10-14 May 1998. http://info.worldbank.org/etools/docs/library/97605/conatrem/conatrem/documents/May98Workshop_Report.pdf

http:/ / dlc.dlib.indiana.edu/dlc/bitstream/handle/10535/304/Mapping_the_ NewCommons.pdf?sequence $=1$

http://www.waterservicesthatlast.org/Resources/Multi-country-synthesis

Recibido el 15 de diciembre de 2012 Aceptado el 12 de noviembre de 2013 